\title{
Prediction of Ore Quantity Based on GA-BP Neural Network
}

\author{
$\mathrm{Li} \mathrm{Guo}^{1}$, Qiong $\mathrm{Wu}^{2 *}$ and Qinghua $\mathrm{Gu}^{2}$ \\ ${ }^{1}$ College of materials \& mineral resource, Xi' an University of Architecture \& Technology, Xi'an, China \\ ${ }^{2}$ School of Management, Xi'an University of Architecture and Technology, Xi'an, Shaanxi, China
}

\begin{abstract}
BP neural network is a multilayer feedforward network trained by error back-propagation algorithm, which is one of the most widely used neural network models. However, BP neural network has exposed more and more shortcomings and deficiencies with the expansion of the application scope. In the prediction of ore quantity, BP neural network has the characteristics of slow convergence and easy to fall into local minimum point. In order to obtain the global optimal solution, and to improve the defects of BP neural network, this paper proposes combination optimization algorithm of genetic algorithm (GA) and BP neural network to improve the speed and accuracy of forecasting the main design flow chart and the analysis of the sort distinguish algorithm are offered, and then some problem in the design and debugging of the algorithm are discussed. On this basis, the GA-BP neural network model is constructed and applied to optimize the initial weights and threshold value of BP neural network. This model choices the floating point coding method to encode the connection weights and thresholds, and divides subjects into several populations. Through the introduction of selection, mutation, crossover, initial weight and other operators, making operational synergies between the various groups. This study selects 30 geological units, 8 quantitative variables $(\mathrm{Pb}, \mathrm{Zn}, \mathrm{Cu}, \mathrm{Mo}, \mathrm{Si}, \mathrm{Ni}, \mathrm{Co}, \mathrm{V})$ and 12 qualitative variables to carry out empirical analysis. Then the simulation of the algorithm is carried out in MATLAB and the parameters are analysed. By normalizing the input samples, 22 groups of observation data are used as the training data for prediction, and the latter 8 groups of observation data are used as the test data to be verified. The results show that when the ore quantity characteristics are not very significant, the model will produce prediction bias. But the improvement of the algorithm increases the efficiency of the function approach capacity of BP neural network and conquer the BP neural network system's instability. It provides an auxiliary guide for ore prediction, which have higher reference value.
\end{abstract}

Keywords: ore quantity, BP neural network, genetic algorithm (GA), prediction

\section{Introduction}

BP neural network can deal with complex situations on information source, such as incomplete, fuzzy, uncertainty, etc. It has the ability of self-learning, which can transform the difficult knowledge acquisition into the variable structure and the self adjustment process. It greatly facilitates the memory and retrieval of information. The learning process of BP neural network is based on learning knowledge and training experience. It makes scientific judgments and decisions on complex issues, and makes an effective prediction and estimation for the unknown.

BP neural network is suitable for the application of prediction, market analysis, system diagnosis and so on. Many scholars have carried on the research to the BP neural network, and used the network to solve some application problems. However, with the expansion of the application scope, BP neural network has exposed some shortcomings and deficiencies. The merits and demerits of the BP neural network is shown in Table 1.

BP neural network is good at local search, but it is easy to fall into local minimum point, the genetic algorithm is good at the global search, but the local search ability is slightly inadequate. The combination of genetic algorithm and BP neural network can be a good complement to each other. Some scholars combine genetic algorithm and BP neural network to establish the prediction and evaluation model for environmental quality assessment, fault diagnosis, time series prediction, risk assessment and other fields. Drawing on the research results of scholars, this study uses genetic algorithm to improve the BP neural network, and applies it to the prediction of ore quantity.

Table 1. The merits and demerits of the BP neural network.

\begin{tabular}{ll}
\hline Merits & Demerits \\
\hline Self-learning ability & Local minimization problem \\
\hline Nonlinear mapping ability & Slow convergence \\
\hline Fault-tolerant ability & Structural choice by experience \\
\hline Generalization ability & Learning and memory are unstable \\
\hline
\end{tabular}

\section{Review of Literature}

The study on the prediction of ore quantity has been carried out for many years. In China, the researchers are mainly based on the mining situation in the region to carry out predictive analysis. In 2002, a comprehensive study of sulfide deposits was carried out by using the method of geology and comprehensive analysis, Zou (2002) studied

* Corresponding Author: Q. Wu, jones1128@126.com, phone: +86 17792311291

Copyright @ 2017 Canamaple Academia Services, http://press.camdemia.ca

DOI: $10.15273 /$ gree.2017.02.015 
the metallogenic geological conditions, geological characteristics, geophysical characteristics, geochemical characteristics, ore genesis and metallogenic mechanism. On this basis, the location prediction of orebody in mining area was forecasted. Luo (2012) proposed a new method of wide spectrum dyke Swarm (WSDS) for target prediction of metal deposit exploration, this study made the Alataw mountain as an example to forecast the target area of exploration. Du (2014) conducted the research on Dongchuan Yinmin iron-copper deposit, the tectonic geometry, material composition and tectonic style of the genetic algorithmbbro intrusions was studied.

In addition, many scholars have carried on the thorough research to the ore quantity forecast by using neural network, such as Cai (1993 and 1994), Pei (2012), Li (2007), Yan (2011) and so on. For example, Yan Jining combines the Kohonen clustering model and BP prediction model, performs cluster analysis of 1444 mineral points in BaoGutu.

On the basis of former research results, the author in the study conducts the optimization research on the neural network.

\section{Optimization Method 3.1 Fundamental principles}

Genetic algorithm can not only optimize the connection weights of BP neural network, but also optimize the network structure and learning rules. But at present, the optimization theory of genetic algorithm on network structure and learning rules is not mature, so this study mainly focuses on the genetic algorithm to optimize the weights and thresholds of BP neural network.

The basic principle of weights and thresholds of BP neural network optimized by genetic algorithm is: The weights and thresholds of BP neural network are coded by using the genetic algorithm to generate the initial population. The fitting error cost function of BP neural network is used as the fitness function of the genetic algorithm, through genetic manipulation and the individual selection, choosing the best chromosomes as weights and thresholds of BP neural network.

\subsection{Main steps to optimize weights and thresholds}

\section{a) Encoding and initial population production}

Binary encoding and float encoding are the most common coding methods, Binary encoding is simple, but not intuitive, and the accuracy is not high; Float encoding has high calculation precision. So, Float encoding method is used to encode the connection weights. The weights and thresholds of BP neural network are concatenated in a certain order to form an array of floating point numbers. This array is a chromosome of genetic algorithm.

The prediction model of ore quantity is based on three layer BP network structure, the number node of input layer, hidden layer and output layer are N, S and M. The length of the code is:

$$
R=N \times S+S \times M+S+M
$$

For the determination of the number $Y$, If the number is too large, it will lead to slow convergence speed; Too small will reduce the accuracy of network training. So, the value of $\mathrm{Y}$ is 20 in this research.

b) Fitness function

The connection weights of the initial population are assigned to the BP network, and the forward propagation of the input signal is used to calculate the error sum squares of the network between the output value and the expected value. Set fitness function is:

$$
f(\mathrm{i})=\sum_{k=1}^{n}\left|\mathrm{y}_{k}-\mathrm{a}_{k}\right|
$$

Combining the evaluation criteria between the genetic algorithm and BP network, the analysis indicates that the smaller the error absolute value leads to the higher the fitness.

\section{c) Genetic manipulation}

In order to realize the evolutionary steps of genetic algorithm, the research is analyzed from the following aspects.

Selection: Using roulette method to selection, the probability that an individual with large probability is selected.

Crossover: Crossover operator is the most important operation. It can produce new individual populations. By expanding the search space, this algorithm can achieve the purpose of global search. Float encoding method is used in this study, so the crossover operator adopts the arithmetic crossover of floating point type. Assume that $\mathrm{x}_{1}$ and $\mathrm{x}_{2}$ are the parent generation of individuals. Then, the offspring produced by the parent generation is:

$$
\left\{\begin{array}{l}
x_{1}^{\prime}=a x_{1}+(1-\mathrm{a}) \mathrm{x}_{2} \\
x_{2}^{\prime}=a x_{2}+(1-\mathrm{a}) \mathrm{x}_{1}
\end{array}\right.
$$

The value of crossover probability is generally between 0.1 and 0.99 . The crossover probability is about 0.3 .

Mutation: The random perturbation of the original gene value was performed by using non uniform mutation, The probability of mutation is between 0.001 and 0.1 .

Generation of new population: new individual populations are generated. Calculate the fitness value to determine whether to achieve cycle times or optimization criteria. If so, then go to the next step, otherwise, continue to cycle genetic operations.

Generation of initial weight: After the genetic algorithm reaches the maximum genetic algebra or the set index, the optimized network connection weight is the decoding value of the best individual in the final population.

\subsection{The GA-BP neural network algorithm}

GA-BP neural network is composed of the following three parts: Firstly, the structure of BP neural network is determined by the steps of 1-2; Secondly, the genetic algorithm is used to optimize the BP neural network by the steps of 3-7; Thirdly, BP neural network training by the steps of 8-10. The algorithm steps of genetic algorithm-BP neural network are shown in Figure 1. 


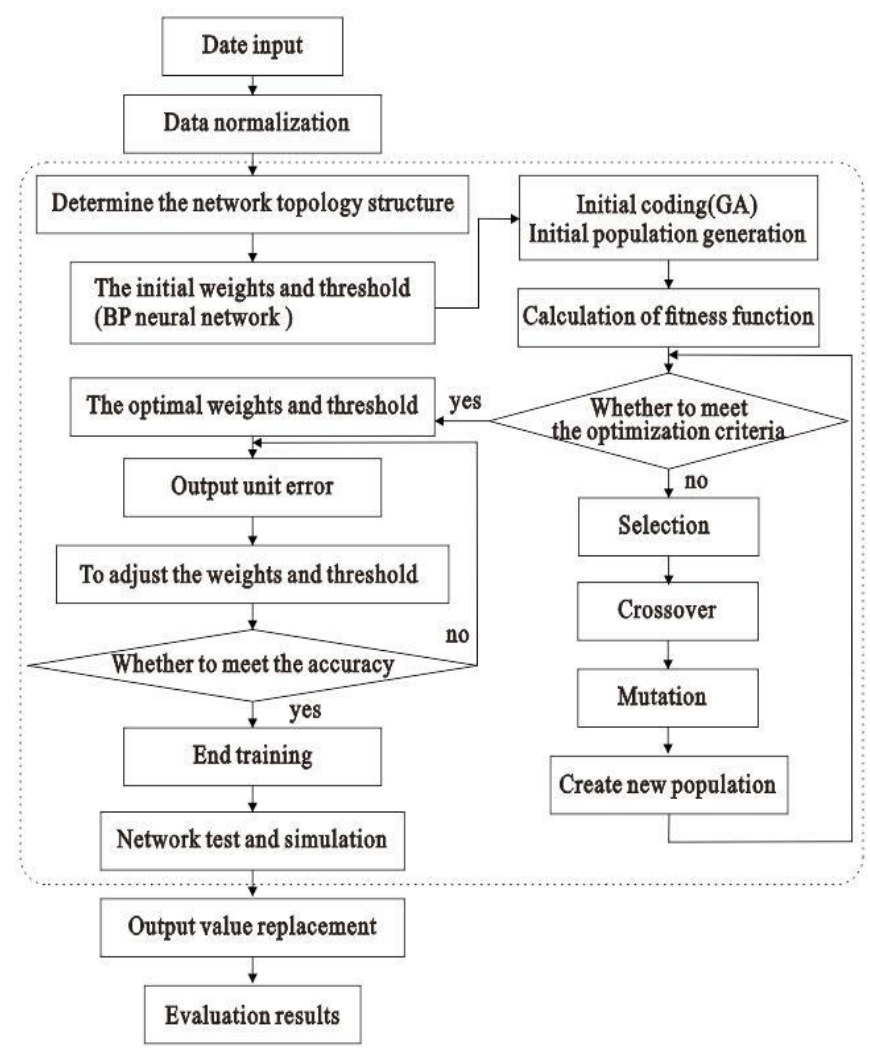

Figure 1. The algorithm steps of GA-BP neural network.

Step 1: To determine the initial structure of BP neural network according to the problem;

Step 2: The BP neural network is trained for a certain number of times to obtain the initial weights and threshold values;

Step 3: Floating point number coding for initial weights and thresholds.

Step 4: The neural network is employed to decide the fitness of the dynamic genetic algorithm. The absolute difference between the output value and the expected value is calculated, and the fitness value $\mathrm{f}(\mathrm{i})$ is calculated according to the absolute difference.

Step 5: To determine whether the individual fitness to meet the optimization criteria, if yes, turn to Step 8; otherwise, turn to the next step.

Step 6: Genetic manipulation. The floating point coding method is proposed to implement GA-BP neural network, and selection operation, cross operation, mutation operation in genetic algorithm are analyzed and improved.

Step 7: Create new group. Repeat Step 3 - Step 6, to determine the initial weights and thresholds until meet the optimization criteria.

Step 8: The optimal solution obtained by genetic algorithm is decoded as the optimal weights and thresholds of BP neural network.

Step 9: BP neural network training.

"Calculation of hidden layer unit and output unit";

"Calculate the output unit error to determine whether to meet the accuracy requirements. If yes, the training ends and turns to Step 10; otherwise, in accordance with the order of BP neural network training";

"Adjust the weights and thresholds of the hidden layer to the output layer, the input layer to the hidden layer";

"Whether it has reached the number of training, if yes, the training ends and turns to Step 10, otherwise, turns to Step 9".

Step 10: End of network training.

\section{Empirical Research 4.1 Parameter setting}

The design of BP network mainly takes into account the number of network layers, the number of neurons in each layer, the transfer function, the network learning algorithm, the initial weights and thresholds.genetic algorithm-BP evaluation model adopts three layer BP neural network structure, Parameter settings for BP - GA is shown in Table 2.

Table 2. Parameter settings for GA - BP.

\begin{tabular}{lll}
\hline \multirow{3}{*}{ BP } & Network structure & $20-10-1$ \\
\cline { 2 - 3 } & Training function & Trainlm \\
\cline { 2 - 3 } & Transfer function of hidden layer & Tansig \\
\cline { 2 - 3 } & Transfer function of output layer & Purelin \\
\cline { 2 - 3 } & Maximum training times & 100 \\
\cline { 2 - 3 } & Target error & 0.001 \\
\cline { 2 - 3 } & Learning rate & 0.01 \\
\hline \multirow{3}{*}{ GA } & Population size & 10 \\
\cline { 2 - 3 } & Chromosome length & 221 \\
\cline { 2 - 3 } & Crossover probability & 0.3 \\
\cline { 2 - 3 } & Mutation probability & 0.01 \\
\cline { 2 - 3 } & Termination generation & 10 \\
\hline
\end{tabular}

\subsection{The GA-BP neural network algorithm}

Geological phenomena are often characterized by some qualitative features, such as the structure environment, formation conditions, rock properties and rock-magma system. These features are significance to the study of geological problems. Through the study on these problems, the study can reveal the various geological processes based on the assessment of present situation and forecast of trend. In order to use GA-BP neural network to predict ore quantity, 30 geological units, 8 quantitative variables $(\mathrm{Pb}$, $\mathrm{Zn}, \mathrm{Cu}, \mathrm{Mo}, \mathrm{Si}, \mathrm{Ni}, \mathrm{Co}, \mathrm{V})$ and 12 qualitative variables, needs to be analyzed and discussed. The original data of geological units are shown in Table 3.

Cs: Lower Carboniferous in Lindi formation and middle Carboniferous in Jingpan Formation;

Tx: Lower Triassic in Xikou formation;

Tw: Upper Triassic in Dalong formation;

Cp: Middle Carboniferous in Huanglong formation, Upper Carboniferous in Chuanshan formation and Lower Permian in Xixia formation;

a: Early Yanshanian monzonitic granite;

b: Early Yanshanian granodiorite;

c: Early Yanshanian granitite;

Fe: The NE trend structures; 
Fc: The NE and EW composite structure;

Tf: Upper Paleozoic fault;

Cs: Contact zone of Lindi formation,Jingpan Formation and granitite;
Ck: Contact zone of Huanglong formation,Chuanshan formation,Xixia formation and granitite.

Table 3. The original data.

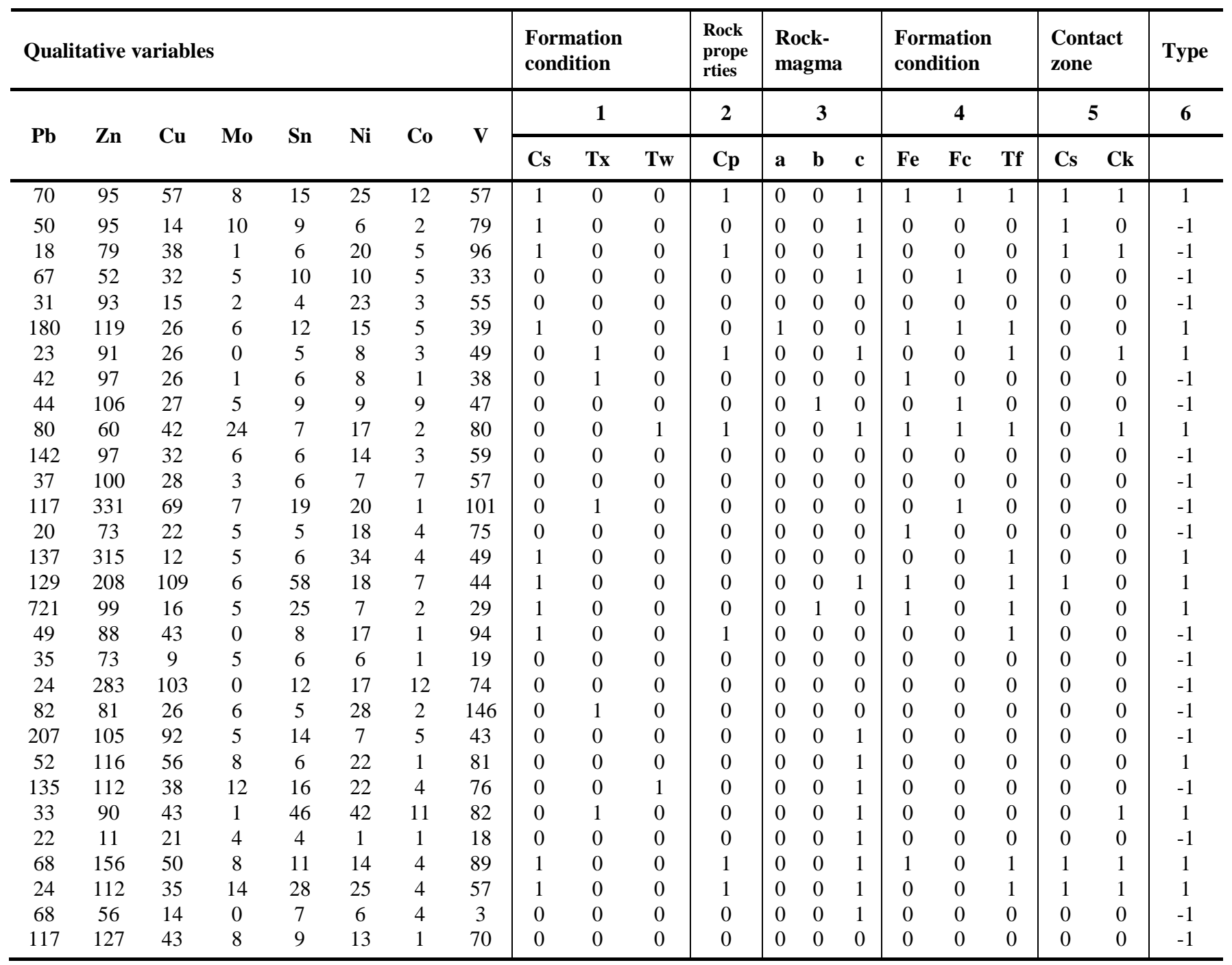

If the original data is too large, the network will easily trap into local minimum points. So, it is necessary to normalize the original data on the basis of an improved neural network training method.

The method of range normalization is used to normalize the output data. The formula for calculation is as follows:

$$
x=\bar{x}^{*}(\max (\mathrm{x})-\min (\mathrm{x}))+\min (\mathrm{x})
$$

\subsection{Experimental results}

As can be seen from Figure 2, The termination algebra of fitness curve is 10 , when the evolution of genetic algorithm to the sixth generation, fitness curve is no longer falling, then the optimal weights obtained by training.

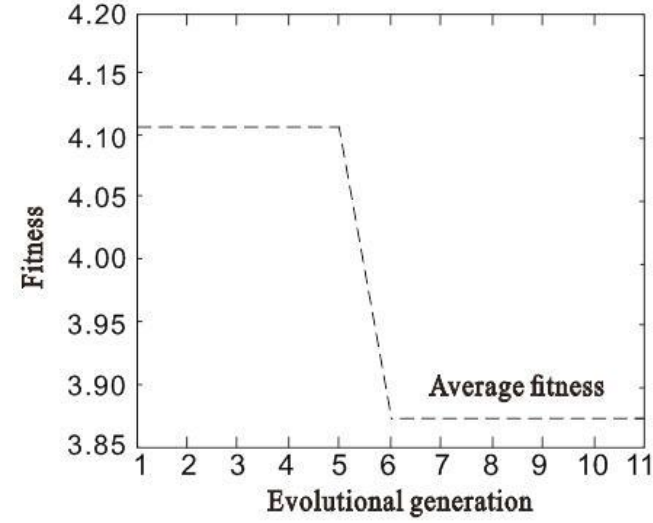

Figure 2. Training results of genetic algorithm model. 
The prediction results are shown in Table 4 . The test

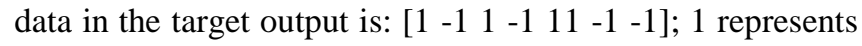
Ore-bearing; -1 represents ore-free.
The interval of the practical system output is: [0.3642 $\begin{array}{lllllll}0.8653 & -1.3196 & -1.0585 & 2.0213 & 1.2127 & -0.8245 & -0.7646]\end{array}$ More than 0 for ore-bearing, less than 0 for ore-free.

Table 4. Prediction results.

\begin{tabular}{|c|c|c|c|c|c|c|c|c|c|c|c|c|c|c|c|c|c|c|c|c|c|}
\hline \multicolumn{8}{|c|}{ Qualitative variables } & \multicolumn{3}{|c|}{$\begin{array}{l}\text { Formation } \\
\text { condition }\end{array}$} & \multirow{2}{*}{ 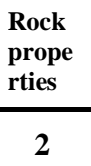 } & \multicolumn{3}{|c|}{$\begin{array}{l}\text { Rock- } \\
\text { magma }\end{array}$} & \multicolumn{3}{|c|}{$\begin{array}{l}\text { Formation } \\
\text { condition }\end{array}$} & \multicolumn{2}{|c|}{$\begin{array}{l}\text { Contact } \\
\text { zone }\end{array}$} & \multirow[t]{2}{*}{$\begin{array}{l}\text { Typ } \\
\text { e }\end{array}$} & \multirow[t]{2}{*}{ Result } \\
\hline \multirow{2}{*}{$\mathbf{P b}$} & \multirow{2}{*}{ Zn } & \multirow{2}{*}{$\begin{array}{l}\mathbf{C} \\
\mathbf{u}\end{array}$} & \multirow{2}{*}{ Mo } & \multirow{2}{*}{$\mathbf{S}$} & \multirow{2}{*}{$\mathbf{N i}$} & \multirow{2}{*}{ Co } & \multirow{2}{*}{$\mathbf{V}$} & \multicolumn{3}{|c|}{1} & & \multicolumn{3}{|c|}{3} & \multicolumn{3}{|c|}{4} & \multicolumn{2}{|c|}{5} & & \\
\hline & & & & & & & & Cs & $\mathbf{T x}$ & Tw & Cp & $\mathbf{a}$ & $\mathbf{b}$ & c & $\mathbf{F e}$ & Fc & $\mathbf{T f}$ & Cs & Ck & & \\
\hline 52 & 116 & 56 & 8 & 6 & 22 & 1 & 81 & 0 & 0 & 0 & 0 & 0 & 0 & 1 & 0 & 0 & 0 & 0 & 0 & 1 & 1 \\
\hline 22 & 11 & 21 & 4 & 4 & 1 & 1 & 18 & 0 & 0 & 0 & 0 & 0 & 0 & 1 & 0 & 0 & 0 & 0 & 0 & -1 & -1 \\
\hline 68 & 156 & 50 & 8 & 11 & 14 & 4 & 89 & 1 & 0 & 0 & 1 & 0 & 0 & 1 & 1 & 0 & 1 & 1 & 1 & 1 & 1 \\
\hline 24 & 112 & 35 & 14 & 28 & 25 & 4 & 57 & 1 & 0 & 0 & 1 & 0 & 0 & 1 & 0 & 0 & 1 & 1 & 1 & 1 & 1 \\
\hline 68 & 56 & 14 & 0 & 7 & 6 & 4 & 3 & 0 & 0 & 0 & 0 & 0 & 0 & 1 & 0 & 0 & 0 & 0 & 0 & -1 & -1 \\
\hline 117 & 127 & 43 & 8 & 9 & 13 & 1 & 70 & 0 & 0 & 0 & 0 & 0 & 0 & 0 & 0 & 0 & 0 & 0 & 0 & -1 & -1 \\
\hline
\end{tabular}

\section{Conclusion}

Through this research, it can be seen that when the characteristics of ore quantity is not very significant, the model will produce prediction bias. This is the systematic deviation of GA-BP neural network algorithm, But the improvement of the algorithm increases the reliability of ore quantity prediction. Combining the genetic algorithm for global search and BP algorithm for local optimization, it can realize the complementary advantages of this two algorithms. The results of the experiment confirmed the GA-BP neural network method is an effective auxiliary method for ore quantity prediction.

\section{Acknowledgement}

This research project is through the discipline talents construction funds support from Xi'an University of Architecture, Technology (RC123), the special fund support from Shaanxi province education department (15JK1390) and the Social Science Foundation of Shaanxi Province(2016R014). The authors also appreciate Prof. S.H. YANG for his assistance in conducting this study.

\section{References}

Cai, Y.D., J.W. Gong and L.S. Yao, 1993. Artificial neural network method for ore prediction, Mineral Resources and Geology, 7(35): 214 - 218.

Cai, Y.D., B. Yang and H. Sun, 1994. Discussion on mineral resource prognostication by self-organization artificial neural network. Hunan Geology, 13(1): 53-55.

Du, Y.L., W.X. Fang, T.R. Wang, Y.Q. Guo and Y.F. Liu, 2014. Characteristics of gabbro-related structures and their prospecting implications in the Yinmin ironcopper deposit in Yunnan. Geotectonica Et Metallogenia, 38(4): 772 - 786.
Li, S.M., S.Z. Yao and Z.G. Zhou, 2007. Application of Matlab based on BP neural network in mineral resource forecast. Metal mine, 374(8): 55 - 57, 67.

Luo, Z.H., B.H. Chen, X.M. Jiang, Z.Q. Wang and Y.H. Wang, 2012. A preliminary attempt for targeting prospecting districts using the wide compositionspectrum dike swarms: A example of the South Alatao Mountains, Xinjiang, China. Acta Petrologica Sinica, 28(7): 1949 - 1965.

Pei, X., 2012. The research of molybdenite mineralization prediction at the Nanping region in Jilin province and Helong city, Ph.D. Thesis, Jilin University.

Yan, J.J., K.F. Zhou, J.L. Wang, H.B. Zhang, C.W. Wan and Z.X. Liu, 2011. Application of artificial neural network to ore-forming prediction. Computer Engineering and Applications, 47(36): 230 - 233.

Zou, H.Y., 2002. Studying on the model of rock-forming and ore-forming, and the prospecting prediction for Karatungk magmatic $\mathrm{Cu}-\mathrm{Ni}$ sulfide deposit in Xinjiang. Ph.D. Thesis, Central South University. 